\title{
THE ROLE OF SPLICING FACTOR SRSF6 IN INCOMPLETE SPLICING OF THE HTT TRANSCRIPT
}

Michael A. Mason ${ }^{1}$, Casandra Gomez Paredes ${ }^{1}$, Aikaterini S. Papadopoulou ${ }^{1}$ and Gillian P. Bates ${ }^{1}$

${ }^{1}$ Huntington's Disease Centre, Department of Neurodegenerative Disease and Dementia Research Institute, Institute of Neurology, Queen Square, London, WC1N 3BG

Background:

Huntington's disease (HD) is caused by an expanded CAG repeat in exon 1 of the HTT gene. In models of HD, it has been shown that an expanded CAG repeat in HTT causes premature termination of HTT RNA during transcription; this occurs by a process called incomplete splicing. Incompletely spliced HTT (HTTexon1) includes exon 1 of the coding region of the HTT gene, as well as a 5' region of intron 1, which is non-coding. HTTexon1 encodes a truncated exon $1 \mathrm{HTT}$ protein, which is implicated in $\mathrm{HD}$ pathogenesis. Although the precise RNA processing mechanism of Httexon1 is unknown, splicing factor SRSF6 has been shown to co-precipitate with transcripts containing Htt intron 1 in HD mice.

Aim:

To elucidate the role of splicing factor SRSF6 in incomplete splicing of $\mathrm{Htt}$ in HD mice.

Methods:

Heterozygous Srsf6 knock out mice $\left(\mathrm{Srsf6}^{+/}\right)$) were generated by CRISPR/Cas9. Characterisation of $\mathrm{Srsf6}^{+/}$- mice was undertaken by quantitative RT-PCR and western blotting. Viability of homozygous Srsf6 knock out $\left(\mathrm{Srsf6}^{-}\right)$mice was examined by inbreeding of $\mathrm{Srsf}^{+/-}$mice. To assess whether decreasing levels of SRSF6 modulates levels of incomplete splicing, Srsf6 ${ }^{+/}$mice were bred to HD knock in mice $(z Q 175)$ and tissues were collected at 2 months of age. Levels of incompletely spliced Httexon 1 were measured by Quantigene, a gene expression assay.

Results:

Srsf6- homozygotes were embryonic lethal, limiting us to the use of Srsf6 ${ }^{+-}$mice only. In Srsf6 ${ }^{+-}$heterozygotes, Srsf6 mRNA was decreased by 50\% in several brain and peripheral regions, and SRSF6 protein was decreased by $70 \%$ in mouse brain compared to wild type mice. However, heterozygosity for Srsf6 knock out did not modulate the level on incomplete splicing in zQ175 mice.

Conclusion:

Ablation of a single Srsf6 allele did not reduce levels of incomplete splicing in HD mice and therefore, further Srsf6 knock down may be required. Accordingly, mouse embryonic fibroblasts (MEFs) have been generated and Httexon1 levels will be measured in HD MEFs after further Srsf6 knockdown by RNA interference. 